\title{
Periodicity due to Coalescence of Atmospheric Pressure Discharge
}

\author{
Takao Fukuyama* and Keisuke Miyazaki \\ Faculty of Education, Nagasaki University, \\ 1-14 Bunkyo-machi, Nagasaki 852-8521, Japan
}

(Received July 7, 2020)

\begin{abstract}
We experimentally investigate the control of atmospheric pressure discharge exhibiting chaotic oscillation. Atmospheric pressure discharge systems, before and during coalescence, are investigated based on photographs, time series, power spectra, and the largest Lyapunov exponents. Through these experiments, it is discovered that the merged discharge system after coalescence shows a periodic oscillation with coherence, and that dynamic behaviors transform from chaotic to orderly.
\end{abstract}

Investigations of various discharge types, such as spark discharge at atmospheric pressure, are important for understanding the property of atmospheric pressure plasma. Considerable effort has been expended in investigating atmospheric pressure plasma ${ }^{1-7)}$ as it is widely applicable in physical sciences research and related technologies. Currently, the control ${ }^{8)}$ of atmospheric pressure discharge is considered an important subject for further study. We use the word control based on the concept of the change of a chaotic trajectory ${ }^{9)}$ to a periodic one. This paper is organized as follows. First, we describe the experimental configuration before and during the coalescence of two discharges. Subsequently, we present the experimental results and discussions concerning dynamic behaviors from the viewpoint of controlling chaotic oscillation. Finally, we summarize the findings.

A schematic configuration of the experimental setup is shown in Fig. 1. Figure 1(a) shows the state where two atmospheric pressure discharges are produced independently. Figure 1(b) shows the process through which the two discharges are merged. A series of experiments was performed at atmospheric pressure using two glass tubes with inner and outer diameters of 9.70 and $12.05 \mathrm{~mm}$, respectively. Argon gas was continuously introduced into each tube at a flow rate of $61.8 \pm 11.12 \mathrm{~mL} / \mathrm{s}$. After applying an ac potential with a high voltage and low frequency to the electrodes, atmospheric Ar discharge was produced. Power supplies A (1220 W, 8.6 kHz, LHV-13AC, LOGY ELECTORIC) and B (276 W, 9.87 kHz, LHV10AC-TL, LOGY ELECTORIC) were used to produce spark discharges. Discharges A and B were 
produced by power supplies A and B, respectively. It is noteworthy that the grounds of discharges A and B were located independently. In this study, we used a device with fixed frequency, voltage, and gas flow rate. The spatiotemporal signals for the analysis were observed along the central axis of the discharges as fluctuations in the light intensity of atmospheric Ar discharge by using a line-scan camera system (TL-4096ACL, TAKENAKA). Spatial signals were sampled as one data point at every $83.3 \mu \mathrm{m}$ interval, and time series were sampled as one data point at every $50 \mu$ s interval. Additionally, a versatile optical camera was used for the measurements.

Figure 2 shows photographs constructed from the measurements of discharges A and B. The left and right portions in Figs. 2(a) and 2(b) correspond to discharges A and B, respectively. Figure 2(a) shows the discharges before coalescence, corresponding to Fig. 1(a). Figure 2(b) shows the discharges during coalescence, corresponding to Fig. 1(b). The total length of the discharge was approximately $8.4 \mathrm{~cm}$ (discharges $\mathrm{A}$ and $\mathrm{B}$ were each approximately $4.2 \mathrm{~cm}$ long). It was discovered that both discharges $\mathrm{A}$ and $\mathrm{B}$ indicated discontinuity in the space at a boundary that separated the two discharges, as shown in Fig. 2(a); by contrast, they were continuous in space, as shown in Fig. 2(b).

Figures 3 and 4 show the time series, power spectra, and orbital correlation diagram of discharges A and B. The time series signal was measured and obtained optically as fluctuations in the light intensity using a line-scan camera; in case of discharge A, the place where $0.8 \mathrm{~cm}$ from the left-hand end of discharge A; in case of discharge $\mathrm{B}$, the place where $0.8 \mathrm{~cm}$ from the right-hand end of discharge B. Figures 3 and 4 (a), (b), and (c) show the signals for discharges $\mathrm{A}$ and $\mathrm{B}$, and the orbital correlation diagram, respectively. Figures 3 and 4 show the state before and during coalescence, respectively. It is noteworthy that the time series data were interpolated using the method presented in a previous study ${ }^{10}$. As shown in Fig. 3, both discharges A and B demonstrate chaotic oscillations, as their power spectra show broad features without prominent sharp peaks. The amplitude shape and fundamental frequency of both discharges differ significantly. The orbital correlation diagram of discharges A and B fills all the regions, as shown in Fig. 3(c). Therefore, it is concluded that the oscillations of the two discharges do not exhibit an orbital correlation. As shown in Fig. 4, discharges $\mathrm{A}$ and $\mathrm{B}$ exhibit periodic oscillations, as their power spectra show features with distinguishable sharp peaks. In particular, the oscillation graphs of both discharges have a prominent peak at $14.2 \mathrm{kHz}$. The orbital correlation diagram of discharges A and B shows their correlation from the linear behavior (see Fig. 4(c)). Therefore, it is concluded that the oscillations of the two discharges exhibit a certain level of orbital correlation. 
The largest Lyapunov exponents before and during coalescence were investigated using the algorithm presented in a previous study ${ }^{11)}$. The exponent has a positive value in the case of chaotic oscillations, and the value increases with the number of chaotic states. Meanwhile, the value approaches zero for a state with periodic oscillations. Before coalescence, the largest Lyapunov exponents exhibited the following values: discharge A, 0.196; and discharge B, 0.578. Meanwhile, during coalescence, they exhibited the following values: discharge A, 0.013; and discharge B, 0.019. The exponents of both discharges decreased and approached zero as a result of coalescence. It was assumed that the degrees of freedom of each chaotic system decreased, and then the complex information concerning change of each amplitude was lost. Hence, the system was controlled, and the periodic orbit was emphasized during coalescence.

The obtained results can be summarized as follows. The two discharges oscillated chaotically with time and exhibited discontinuity in space at the boundary separating them, before the coalescence occurred. However, they oscillated with continuity in space during coalescence. The oscillations of the two discharges indicated a certain level of orbital correlation caused by coalescence. The largest Lyapunov exponents of the two discharges approached zero owing to coalescence. The oscillations of the two discharges transformed from chaotic to orderly as a result of their coalescence.

This study is partly supported by JSPS KAKENHI Grant Number JP20K03895.

\footnotetext{
*E-mail: fukuyama-takao@nagasaki-u.ac.jp
} 
1) S. Kanazawa, M. Kogoma, T. Moriwaki, and S. Okazaki, J. Phys. D: Appl. Phys. 21, 838 (1988).

2) S. Okazaki, M. Kogoma, M. Uehara, and Y. Kimura, J. Phys. D: Appl. Phys. 26, 889 (1993).

3) D. Trunec, A. Brablec, and F. Stastny, Contrib. Plasma Phys. 38, 435 (1998).

4) D. Trunec, A. Brablec, and J. Buchta, J. Phys. D: Appl. Phys. 34, 1697 (2001).

5) G. S. Selwyn, H. W. Herrmann, J. Park, and I. Henins, Contrib. Plasma Phys. 6, 610 (2001).

6) M. Teschke, J. Kedzierski, E. G. Finantu-Dinu, D. Korzec, and J. Engemann, IEEE Trans. Plasma Sci. 33, 310 (2005).

7) T. Fukuyama, K. Ota, and H. Sakamoto, Phys. Plasmas 25, 092303 (2018).

8) T. Fukuyama, K. Hagimine and R. miyazaki, J. Phys. Soc. Jpn. 86, 095003 (2017).

9) M. Ohno, M. Tanaka, A. Komori, and Y. Kawai, J. Phys. Soc. Jpn. 58, 28 (1989).

10) H. Akima, J. Assoc. Comput. Mach. 17, 589 (1970).

11) A. Wolf, J. B. Swift, H. L. Swinney, and J. A. Vastano, Physica D 16, 285 (1985). 
(a)

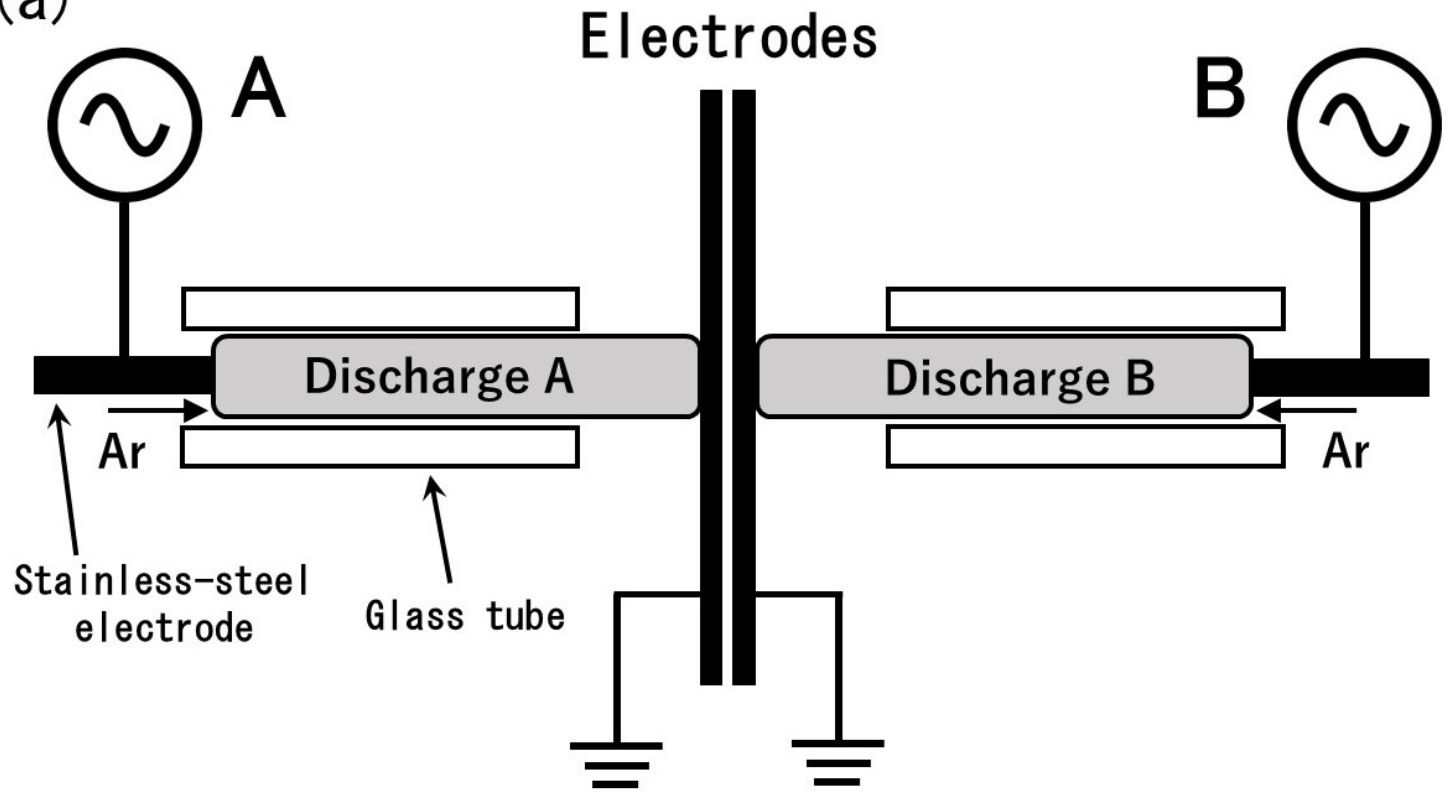

(b)

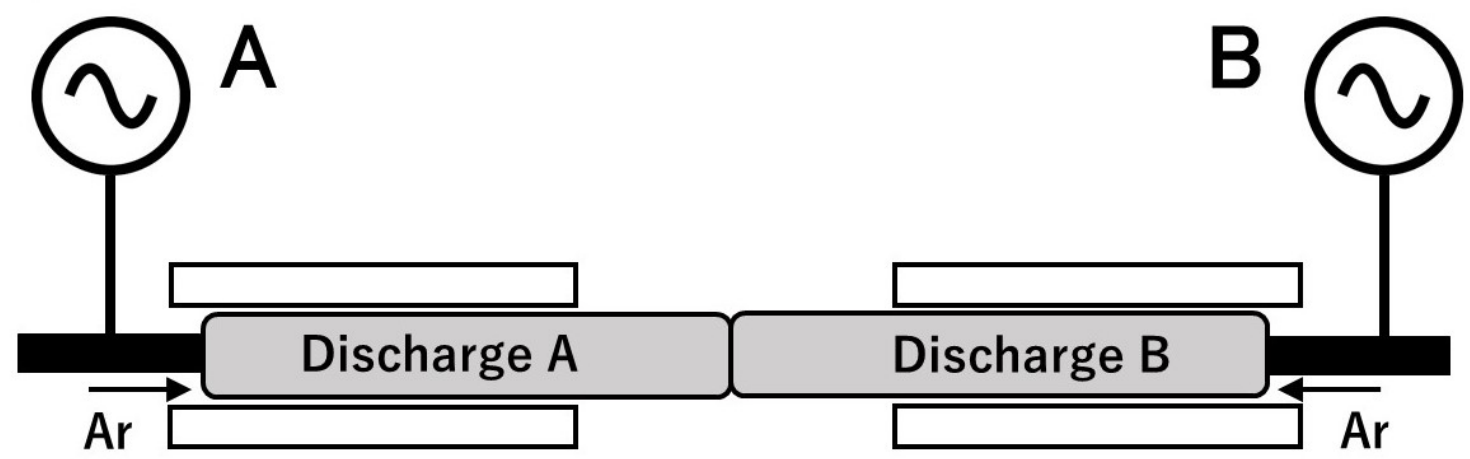

Fig. 1. Schematic configuration of experimental setup: (a) State where two atmospheric pressure discharges are produced independently; (b) state where two discharges merged. 
(a)
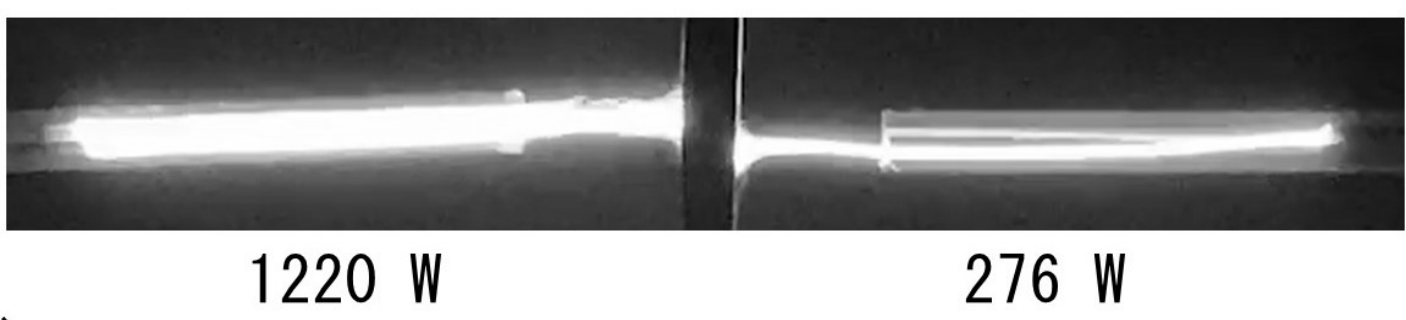

(b)

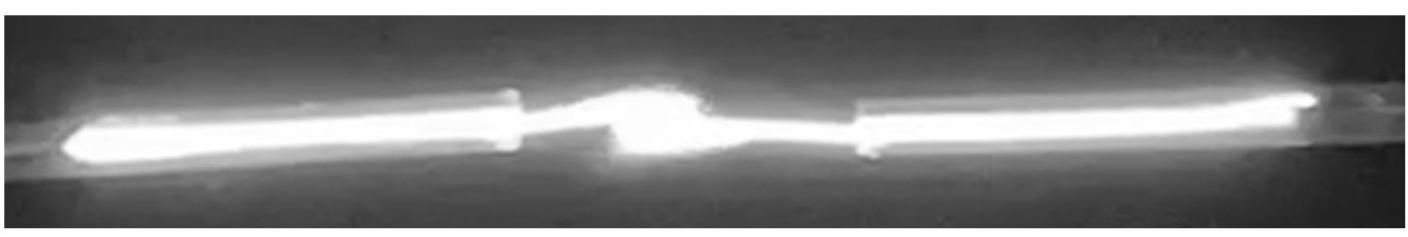

Fig. 2. Photographs of discharges $A$ and B. Left and right panel correspond to discharges A and B, respectively: (a) State in which discharges A and B are produced independently before coalescence, corresponding to Fig. 1(a); (b) state during coalescence, corresponding to Fig. 1(b).

(a)
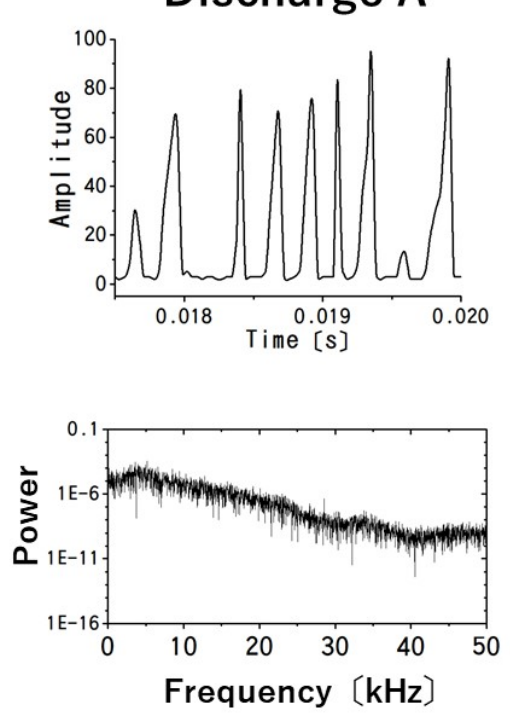

(b) Discharge B
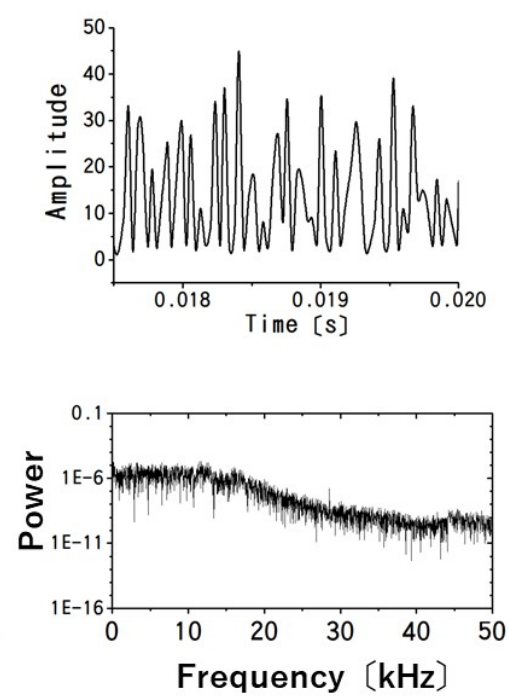

(c)

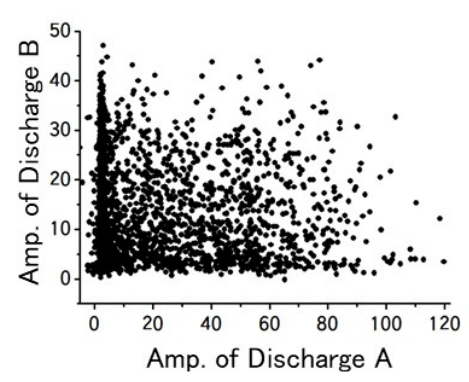

Fig. 3. Time series, power spectra, and orbital correlation diagram, before coalescence: (a) Discharge A; (b) discharge B; (c) orbital correlation diagram of discharges A and B. 
(a)
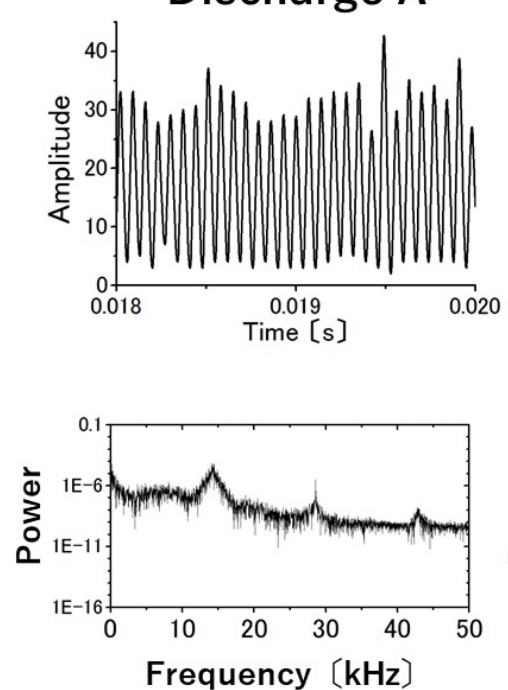

(b) Discharge B
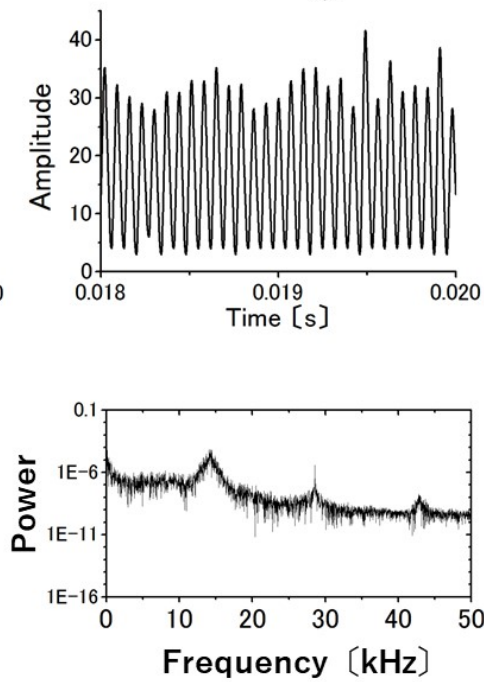

(c)

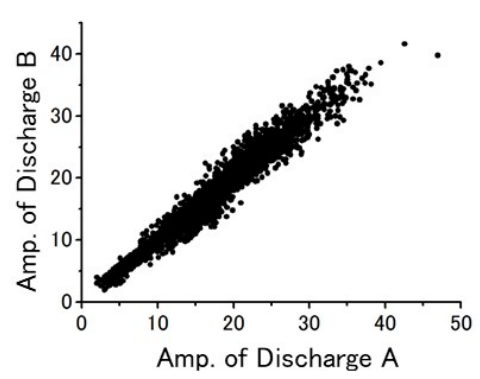

Fig. 4. Time series, power spectra, and orbital correlation diagram, during coalescence: (a) Discharge A; (b) discharge B; (c) orbital correlation diagram of discharges A and B. 